\title{
A influência das agendas governamentais na produção multidisciplinar de conhecimento
}

\section{Christiana Freitas*}

Fernanda Sobral*

\begin{abstract}
Resumo A política científica e tecnológica recente indica a tendência à adoção de um modelo de desenvolvimento científico e tecnológico misto, no qual práticas acadêmicas e políticoinstitucionais tradicionais entrelaçam-se a novas. $\mathrm{O}$ modelo misto articula a demanda espontânea e a demanda induzida, a comunidade científica, o governo, setores empresariais e outros interessados no desenvolvimento científico e tecnológico. Esse modelo procura associar a lógica do campo científico, ou seja, as demandas da ciência às demandas econômicas e sociais, permitindo a articulação entre os campos mencionados. Percebe-se, com isso, a fluidez de fronteiras entre disciplinas sem que as diferenças deixem de existir. O artigo apresentado tem como objetivo refletir sobre a política científica e tecnológica em questão, focando mais especificamente no papel das agendas governamentais no estímulo ao desenvolvimento da produção do conhecimento multidisciplinar no Brasil. A análise do Plano Plurianual do Ministério da Ciência e Tecnologia (PPA-MCT) e das diretrizes e estratégias adotadas por algumas agências de fomento à pesquisa do Brasil, como a Capes e o $\mathrm{CNPq}$, revelou estímulo desses programas à expansão e à consolidação da multidisciplinaridade. Concluímos que existem alguns mecanismos institucionais que terminam por estimular, propositadamente ou não, a multidisciplinaridade no processo de produção do conhecimento científico-tecnológico.
\end{abstract}

Palavras-chave modelo misto de desenvolvimento científico e tecnológico, multidisciplinaridade, agendas governamentais.

\begin{abstract}
Recent scientific and technological policies indicate a tendency to incorporate a mixed model of science production. In this model, traditional academic, political and institutional practices appear to blend with new ones. In such mixed model spontaneous and induced demands for research are closely related. Moreover, various social spheres are integrated with the scientific community, such as the government agencies, the business sector and other areas engaged in the development of science and technology. This model associates different logics and goals by mixing social, economic and scientific demands. Fluidity among frontiers is obvious as they maintain, simultaneously, their differences. The main goal of this article is to reflect upon the recent scientific and technological policies mentioned above, focusing more specifically on the role of government agendas in stimulating the development of multidisciplinary knowledge production in Brazil. The analysis of the Pluriannual Plan of the Ministry of Science and Technology of Brazil (PPA-MCT) and of the guidelines and strategies adopted by some of the government development agencies such as Capes and CNPq has revealed that those programs provide significant stimulus to the expansion and consolidation of multidisciplinarity. This observation leads to the conclusion that there are some institutional mechanisms that encourage - intentionally or not - multidisciplinarity in the science and technology knowledge production process.
\end{abstract}

Doutora em Sociologia, pesquisadora do Núcleo de Estudos sobre Ensino Superior no Brasil - NESUB/UnB

.* Doutora em Sociologia, Professora do Departamento de Sociologia da UnB 
Keywords mixed model of scientific and technological development; multidisciplinarity; governmental agendas.

\section{Introdução}

Enquanto todos se encantam com o crescimento vertiginoso da Internet e com as possibilidades de comunicação e interação quase infinitas nos mais variados modelos de aparelhos celulares, transformações mais sutis ocorrem nas diferentes esferas da sociedade. O período da "revolução" tecnológica e informacional, iniciado na década de 1990, parece ter chegado ao fim. Vivemos agora a "era" tecnológica e informacional, quer o mundo queira quer não, quer o mundo todo participe dessa era ou não. A proposta deste artigo é tecer algumas considerações a respeito das transformações no papel do conhecimento e da informação, com o foco central na expansão da produção multidisciplinar do conhecimento observada na contemporaneidade.

A informação passa a ser mais acessível. Até a economia recebeu um nome composto: "economia do conhecimento". Ao que parece, o conhecimento passou a ser indispensável para tudo e para todos. Não apenas a sociedade se transforma com o conhecimento que a penetra de formas cada vez mais sutis e profundas. O próprio conhecimento vem experimentando a sua gênese, a sua transformação com base não apenas nele mesmo, mas com base, também, naquilo que a sociedade vem fazendo dele. O conhecimento é transformado, ao mesmo tempo em que se transforma.

Durante a primeira metade da década de 1990, sociólogos da ciência apontaram características que constituiriam um "novo modo de produção do conhecimento" (GIBBONS et al., 1994). Uma das tendências marcantes associadas a esse novo modelo de produção, inclusive em domínios acadêmicos, é à construção de artefatos destinados à comercialização. Têm aplicação imediata e são construídos visando à solução, preferencialmente rápida, de problemas já existentes. É a ciência que se faz em um contexto de aplicação. Muitas vezes tais artefatos têm o conhecimento científico como objeto central de sua constituição. É o caso de vários projetos elaborados no campo das ciências da computação, incluindo aí a Inteligência Artificial. A intenção é criar programas com a meta de reutilização, reorganização e "remodelamento" de conceitos (como é o caso da área de knowledge modeling ou modelamento do conhecimento), sugerindo uma reconstrução de idéias e teorias científicas já existentes. Essa reconstrução acontece não apenas em áreas da computação, mas permeia todo o campo de produção do conhecimento (FREITAS, 2003). Os conceitos não são separados de acordo com sua "procedência disciplinar", mas incorporados à teoria desenvolvida de acordo com a necessidade do momento, que pode ser tanto a produção de um artefato tecnológico quanto a solução de um problema que foi incorporado à agenda acadêmica a partir de demandas sociais.

Recentemente, alguns autores aprimoraram as análises para chegar à conclusão de que existe, hoje, não necessariamente um novo modo de produção de conhecimento, mas sim o surgimento de um "modelo misto de desenvolvimento científico e tecnológico", impulsionado pelas demandas da própria ciência e, ao mesmo tempo, pelas demandas econômicas e sociais (SOBRAL; TRIGUEIRO, 1994). Este modelo incorpora práticas inovadoras àquelas tradicionalmente estabelecidas na academia, particularmente a questão da multidisciplinaridade objeto central deste trabalho. Duas características desse modelo são fundamentais para o estímulo à multidisciplinaridade: o contexto de aplicação e a demanda econômica e social que interferem nessa produção. O conhecimento não é orientado apenas para os pares, mas também para os não produtores de conhecimento, implicando maior responsabilidade social com relação 
ao que é produzido. Nos anos mais recentes, observou-se um aumento significativo da consciência pública sobre os mais variados temas que atingem a população, diretamente ou não. Meio ambiente, saúde, novas tecnologias reprodutivas passam a ser temas correntes entre cidadãos. As transformações vividas no campo da ciência foram especialmente estimuladas por movimentos sociais que, muitas vezes por intermédio da imprensa e de organizações nãogovernamentais, exigiam mais transparência no processo de desenvolvimento de pesquisas. Uma das exigências da sociedade civil que atestou essa tendência foi a de que os custos com a produção de conhecimento fossem explicitados e revelados em fóruns públicos.

Autores como Nowotny, Scott e Gibbons (2001) apontam para a tendência atual de produção de conhecimento voltado para a solução de problemas que são, muitas vezes, frutos de demandas sociais. A solução para esses problemas, por sua vez, acontece a partir da colaboração de diversas áreas do conhecimento. A ciência, antes produtora de resultados que somente depois de prontos passavam para o domínio da sociedade, hoje lida com demandas dessa sociedade. Muitas vezes, são essas demandas que irão orientar a produção científica e tecnológica (NOWOTNY; SCOTT; GIBBONS, 2001).

Além disso, o contexto atual pressupõe heterogeneidade institucional. Envolve várias organizações, entre as quais empresas de diferentes portes, empresas de redes, de alta tecnologia, laboratórios de pesquisa, organizações não-governamentais, bem como programas de cooperação nacional e internacional de pesquisa. Há um aumento do número de locais onde o conhecimento passa a ser produzido, além da diversificação observada das formas de estabelecimento de redes de comunicação. Essa heterogeneidade institucional gera confluência e entrelaçamento de linguagens, termos, conceitos, favorecendo também a multidisciplinaridade. A partir da análise de três setores da sociedade - governo, empresas e instituições de ensino superior - essa heterogeneidade fica patente. Segundo dados do Ministério da Ciência e Tecnologia, o ensino superior ainda é o setor que mais possui pessoas envolvidas com pesquisa e desenvolvimento. Entretanto, o gráfico abaixo mostra a tendência, já observada, ao envolvimento crescente de outros setores da sociedade com o desenvolvimento de pesquisas nas mais diferentes áreas do conhecimento. É o que acontece no setor privado sem fins lucrativos e nas empresas. 


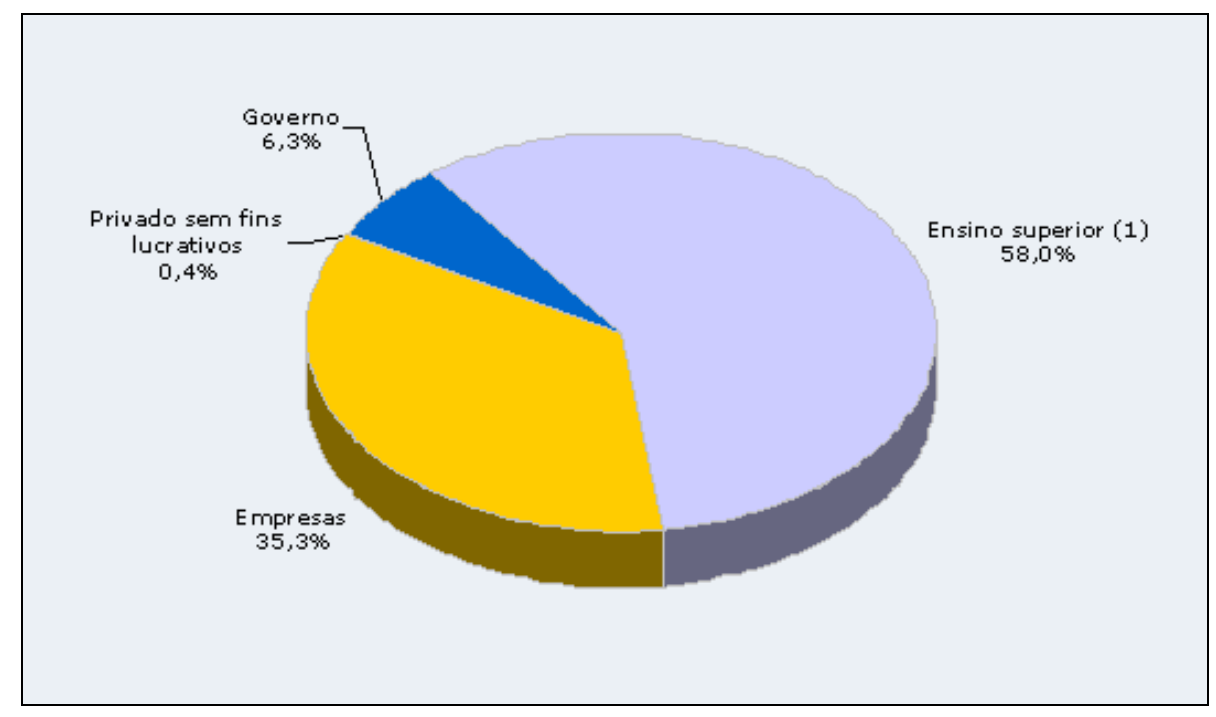

Figura 1: Brasil - Percentual de pessoas envolvidas em pesquisa e desenvolvimento (P\&D) em equivalência de tempo integral, por setor institucional, 2000

Fontes: para as empresas: Pesquisa Industrial de Inovação Tecnológica (Pintec) - 2000, do Instituto Brasileiro de Geografia e Estatística (IBGE), para os estudantes de doutorado: Fundação Coordenação de Aperfeiçoamento de Pessoal de Nível Superior (Capes) e, para os outros: Diretório dos Grupos de Pesquisa no Brasil (DGP), Censo 2000, da Assessoria de Estatística e Informação (AEI), do Conselho Nacional de Desenvolvimento Científico e Tecnológico (CNPq) ${ }^{1}$. Elaboração: CoordenaçãoGeral de Indicadores - Ministério da Ciência e Tecnologia.

O modelo misto de produção do conhecimento faz convergir, muitas vezes, diversos objetos de estudo, conceitos e aportes teóricos. O conhecimento que abarca diferentes pontos de vista, conceitos e parâmetros ocorre não apenas entre campos distintos da sociedade, mas também se desenvolve no interior do campo de produção científico-tecnológica, estimulando o desenvolvimento da multidisciplinaridade, hoje parte do quotidiano acadêmico de produção. Essa situação não se verifica apenas no Brasil, mas abrange a produção do conhecimento de forma geral. Pesquisas recentes demonstram como descobertas em uma disciplina ou área do conhecimento influenciam a produção do conhecimento em outras áreas, gerando conhecimento fruto de convergências temáticas entre disciplinas. Diferentes áreas da ciência trabalham conjuntamente, visando ao desvendamento de fenômenos, soluções ou conceitos específicos, produzindo o conhecimento multidisciplinar.

Vários fatores podem ser apontados como estímulos a esse contexto. Cientistas em Porto Rico realizaram estudos com o intuito de identificar possíveis relações entre a multidisciplinaridade verificada entre grupos de pesquisa e a produção de novo conhecimento (SANZ-CASADO et al., 2004). A conclusão foi que existe, de fato, influência de práticas multidisciplinares na construção de conceitos, temas e práticas acadêmicas na atualidade. Mas o que, por sua vez, vem estimulando a prática multidisciplinar?

Este artigo irá focar em alguns dos mecanismos existentes que terminam por estimular, propositadamente ou não, o desenvolvimento da produção multidisciplinar de conhecimento. Serão aqui ressaltados os mecanismos relativos às agendas governamentais no Brasil. A análise de instrumentos governamentais de planejamento, como o Plano Plurianual do Ministério da

\footnotetext{
${ }^{1}$ Pode haver dupla contagem, devido ao fato de uma pessoa estar envolvida em pesquisa e desenvolvimento (P\&D) em mais de um setor institucional; foram excluídos os pesquisadores que não informaram a titulação máxima e os estudantes que não informaram o nível de treinamento, no Diretório dos Grupos de Pesquisa no Brasil (DGP); Os estudantes cadastrados no Diretório dos Grupos de Pesquisa no Brasil (DGP) foram colocados no setor de Ensino Superior.
} 
Ciência e Tecnologia (PPA-MCT), ${ }^{2}$ as ações temáticas de fomento do CNPq e os cursos de pósgraduação oferecidos pela Capes, além de alguns programas do MCT, revela estímulo significativo desses programas à expansão e à consolidação da multidisciplinaridade.

O objetivo deste artigo, como sugerido, é ressaltar o papel das agendas governamentais no estímulo ao desenvolvimento da multidisciplinaridade no processo de produção do conhecimento no Brasil. Interessa, principalmente, perceber como as agendas governamentais vêm favorecendo o desenvolvimento da multidisciplinaridade. $\mathrm{O}$ conceito de multidisciplinaridade refere-se aqui, principalmente, à relevância de questões de pesquisa de uma área do conhecimento que também são tidas como importantes para outra; evoca a noção de "intersetorialidade". Vários grupos de pesquisa, pertencentes a diferentes setores da ciência e da sociedade, participam do desenvolvimento de produtos voltados, geralmente, para a aplicabilidade. Tais produtos orientam-se com base em temas, que tendem a aglutinar grupos, objetivos e conceitos diferentes. Todos esses elementos distintos e, muitas vezes, discordantes, unem-se, visando ao desenvolvimento e à conclusão de um projeto em foco. Vale ressaltar que o trabalho multidisciplinar não resulta, necessariamente, em um novo conceito ou aporte teórico. A multidisciplinaridade diz respeito à reunião de elementos de inúmeras áreas do conhecimento com a finalidade de alcançar determinada meta que, na maioria das vezes, não é a produção de elementos cognitivos totalmente novos, frutos daquela união.

Projetos desenvolvidos por pesquisadores nas mais diferentes instituições acadêmicas não pertencem, necessariamente, a apenas uma área do conhecimento ou a apenas um setor da sociedade. Em pesquisa recente, um periódico eletrônico e sua rede de pesquisadores foram analisados (FREITAS, 2003). O periódico eletrônico, resultado da produção de um artefato tecnológico específico, foi desenvolvido numa instituição de ensino e pesquisa, financiado pelo governo britânico e algumas empresas interessadas no projeto. Pertence à área de educação, mas apresenta inúmeras interfaces com a área de visualização de software, já que esse veículo de comunicação utiliza um software específico que permite um tipo de imagem na tela antes indisponível.

Esse fenômeno de "descompartimentalização", observado atualmente nos institutos de pesquisa, foi notado por um dos integrantes da rede estudada. Este comentou que há cinco ou seis anos atrás, as pessoas com backgrounds diferentes não se comunicavam e que a antiga "compartimentalização" tende a não existir mais. A multidisciplinaridade, apontada como uma das características do modelo misto de desenvolvimento científico e tecnológico, pode ser aqui constatada como parte integrante das práticas e relações acadêmicas contemporâneas. A rede acadêmica envolvida com o projeto estudado retrata esse contexto atual (FREITAS, 2003). Cada um dos cinco principais integrantes vem de uma disciplina distinta. Segundo outro pesquisador, também integrante da rede, essas diferenças são, muitas vezes, positivas: "basicamente, temos dois tipos de dia. Num mau dia nós não entendemos um ao outro. Num bom dia, você tem essa criatividade fantástica, resultado de termos reunido pessoas com idéias e perspectivas diferentes" 3 .

A seguir, será apresentada a análise de alguns fatores institucionais que estimulam o convívio de universos tão diferentes, que terminam por cooperar um com o outro visando ao desenvolvimento de objetivos e temas comuns.

\footnotetext{
2 O Plano Plurianual pode ser acessado em: http://www.mct.gov.br/comunic_old/agend214.htm.

${ }^{3}$ Entrevista realizada em 27 de maio de 2002 para pesquisa de campo da tese de doutorado intitulada Práticas sociais no ciberespaço: as redes de organização e distribuição do conhecimento científico-tecnológico (FREITAS, 2003).
} 


\section{A multidisciplinaridade no fomento do CNPq}

Interessante notar, em primeiro lugar, que a tendência à multidisciplinaridade na produção científica não ocorre em todas as áreas do conhecimento da mesma forma e com a mesma intensidade. Enquanto algumas áreas do conhecimento desenvolvem trabalhos multidisciplinares há um certo tempo, outras ainda adotam práticas tradicionais relacionadas ao modo de produção do conhecimento existente antes do rápido desenvolvimento das tecnologias da informação e das transformações delas advindas (FREITAS, 2003). As diferenças entre áreas do conhecimento no tocante à multidisciplinaridade foram apontadas em estudo realizado, em 2003, pelo Núcleo de Pesquisa sobre Ensino Superior da Universidade de Brasília (SOBRAL; MARTINS; VELLOSO, 2003). O objetivo da pesquisa foi identificar possíveis convergências entre resultados de ações de fomento do $\mathrm{CNPq}$ e as prioridades temáticas estipuladas pelo Plano Plurianual de Ações (PPA) do Ministério da Ciência e Tecnologia (MCT - de 2000 a 2003). ${ }^{4}$ A intenção da pesquisa, portanto, foi analisar a produção do conhecimento sob o prisma da afinidade - ou não - dessa produção com as prioridades governamentais. Apesar de o objetivo central da pesquisa não ter sido a identificação da multidisciplinaridade nas áreas do conhecimento analisadas, essa característica foi observada em diversas situações.

Não nos interessa expor, na íntegra, a análise já feita pela pesquisa citada, qual seja a observação detalhada da relação de convergência entre o fomento do $\mathrm{CNPq}$ à pesquisa e as prioridades temáticas estipuladas pelo Plano Plurianual (PPA) do Ministério da Ciência e Tecnologia (MCT: 2000-2003). O que interessa neste ensaio, como já apontado na introdução, é perceber como as agendas governamentais vêm favorecendo o desenvolvimento da multidisciplinaridade. A partir dessa tese central, explicitamos os mecanismos que geram o contexto multidisciplinar de produção do conhecimento em áreas de fomento do $\mathrm{CNPq}$, buscando apontar as características desse fenômeno. Ao que tudo indica, as agendas governamentais, tais como aquelas adotadas pelo CNPq e pela Capes, vêm de fato estimulando a produção de conhecimento multidisciplinar.

Cabe apresentar, aqui, algumas conclusões significativas da pesquisa para que se possa, posteriormente, analisar questões relacionadas à multidisciplinaridade. Uma das conclusões centrais obtidas com a pesquisa mencionada revela a convergência de resultados de fomento do $\mathrm{CNPq}$, a partir da demanda espontânea, com determinadas prioridades temáticas estipuladas pelo PPA, principalmente em algumas grandes áreas do conhecimento, tais como as Ciências

\footnotetext{
4 Foram considerados como resultados de ações de fomento do CNPq: os relatórios de pesquisa de bolsistas do Programa Institucional de Bolsas de Iniciação Científica - PIBIC, elaborados em 2000, as dissertações de mestres ex-bolsistas do CNPq e as teses de doutores que foram bolsistas do CNPq, defendidas em 2000 e os projetos de pesquisa de bolsistas de produtividade que receberam bolsa em 2000 nas diferentes áreas de conhecimento, ou seja, Ciências Agrárias, Ciências Biológicas, Ciências Exatas e da Terra,Ciências Humanas, Ciências da Saúde, Ciências Sociais Aplicadas, Engenharias,Lingüística e Letras. Como prioridades temáticas foram tratadas questões, temas, objetivos ou metas, ou ações expressas nos documentos relativos aos programas temáticos prioritários definidos pelo PPA-MCT para o período: Aplicações Nucleares na Área Médica, Biotecnologia e Recursos Genéticos, C\&T para Agronegócios, C\&T para Ecossistemas; Climatologia, Meteorologia e Hidrologia; Desenvolvimento Tecnológico para a Área Nuclear, Fomento ao Desenvolvimento Tecnológico no Setor Petrolífero, Fomento à Pesquisa em Saúde, Mudanças Climáticas, Nacional de Atividades Espaciais, Produção de Equipamentos para a Indústria Pesada, Segurança Nuclear e Sociedade da Informação. Assim, o ponto de partida das eventuais convergências identificadas era a demanda espontânea, não induzida, apoiada pela agência em anos anteriores à vigência do PPA/MCT embora algumas dessas prioridades já fossem objeto de ações por parte do CNPq, antes de serem incorporadas pelo PPA. Deve-se ressaltar que embora a análise dos resultados do fomento , através da medição da produção científica e das citações ,seja uma tradição importante nos estudos de C \& $\mathrm{T}$, essa pesquisa inovou ao analisar a produção sob o prisma de verificar a sua afinidade com as prioridades governamentais. Para isso, os vários consultores analisaram um total de aproximadamente 6500 resumos que foram enquadrados ou não nessas prioridades.
} 
Agrárias, Ciências da Saúde e Ciências Biológicas. Além disso, os dados indicaram também que nessa convergência destacaram-se determinados programas, tais como Ciência e Tecnologia (C\&T) para Agronegócios, Fomento à Pesquisa em Saúde, Biotecnologia e Recursos Genéticos e, finalmente, Sociedade da Informação.

Deve-se ressaltar que a origem desses programas encontra-se vinculada a ações de fomento desenvolvidas pelo CNPq antes mesmo da elaboração do PPA. Alguns deles tiveram início ainda na década de 1980, como o PRONAB (Programa Nacional de Biotecnologia), o PADCT (Programa de Apoio ao Desenvolvimento Científico e Tecnológico) e o RHAE (Programa de Formação de Recursos Humanos em Áreas Estratégicas). Já no PADCT a multidisciplinaridade era visível, uma vez que incluía entre seus subprogramas os de Biotecnologia, Ciências Ambientais, Informação em Ciência e Tecnologia e o RHAE (Programa de Formação de Recursos Humanos em Áreas Estratégicas). Vale ressaltar que os subprogramas já revelavam característica importante e atual que estimulava significativamente a multidisciplinaridade: baseavam-se em temas, que congregavam uma série de áreas disciplinares específicas.

Em 1995, o planejamento estratégico do CNPq definiu Saúde, Educação, Informática, Meio Ambiente e Agricultura como temas prioritários de atuação. Desde 1991, a área de Saúde começou a discutir a política de ciência e tecnologia em saúde, culminando, em 1994, com a I Conferência Nacional de Ciência e Tecnologia em Saúde e, em 1997, com o programa de Indução Estratégica à Pesquisa em Saúde. A área de Informática, que já estava presente no PADCT e no RHAE, recebeu também incentivos de outros programas, destacando-se o DESIBRA (Programa de Desenvolvimento Estratégico em Informática no Brasil).

Os temas que mais revelaram convergência de resultados de fomento do $\mathrm{CNPq}$ com as prioridades temáticas do PPA foram, não coincidentemente, temas em que se constatou uma expressiva transversalidade, intersetorialidade ou multidisciplinaridade. Foi o caso, por exemplo, das Ciências Biológicas, que desenvolvem pesquisas ligadas a temas como Biotecnologia e Recursos Genéticos, Saúde e Agronegócios. As Ciências da Saúde também tratam de temas que favorecem a multidisciplinaridade, reunindo áreas como a Biotecnologia, Recursos Genéticos e Sociedade da Informação. Uma questão ou tema de pesquisa de uma grande área passa a ser importante, também, para outras áreas ou disciplinas. Exceção é o caso das Ciências Agrárias. Embora tenham apresentado as maiores porcentagens de convergência com os temas prioritários do Plano Plurianual, os dados também mostram que um único programa praticamente absorveu todas as pesquisas das Ciências Agrárias (C\&T para Agronegócios), ou seja, observou-se grande concentração das pesquisas dessa grande área numa única temática. Pode-se observar, apesar da exceção apontada, a tendência à multidisciplinaridade em áreas do conhecimento que desenvolvem pesquisas e trabalhos comuns em torno de um tema que as agrega ou reúne. Ou seja, os temas tratados e indicados pelas agendas governamentais como prioritários contribuem, direta ou indiretamente, para a produção multidisciplinar do conhecimento, uma vez que tais temas contemplam várias disciplinas.

Já nas Ciências Humanas e nas Ciências Exatas e da Terra, as pesquisas se enquadraram prioritariamente em dois programas - Sociedade da Informação e Fomento à Pesquisa em Saúde. É interessante notar que duas áreas diferentes convergiram com os mesmos programas, embora não nos mesmos temas. A inserção das Ciências Exatas e da Terra no Programa Sociedade da Informação parece se dever, sobretudo, à presença da área de Ciência da Computação. Já a Química revelou-se como a principal responsável pela participação da grande área no Programa de Fomento à Pesquisa em Saúde, dada a pesquisa em qualidade e produção de imunobiológicos e medicamentos. 
As Ciências Humanas participaram do programa Sociedade da Informação, principalmente do tema "Educação na Sociedade da Informação". As Ciências Sociais Aplicadas, Letras e Lingüística convergiram principalmente com relação a um único programa, o da "Sociedade da Informação". Nas Grandes Áreas de Letras, Lingüística e Engenharias, os graus de convergência foram inexpressivos, mesmo nos programas que apresentaram maior enquadramento: Sociedade da Informação e Produção de Equipamentos para a Indústria Pesada. Ao mesmo tempo em que foi registrada quase nenhuma convergência entre as pesquisas financiadas pelo CNPq nessas áreas específicas com os programas temáticos prioritários do PPA, a multidisciplinaridade também aparece de forma inexpressiva, sendo observada em proporções bastante reduzidas quando comparadas ao contexto de produção multidisciplinar das outras áreas do conhecimento.

Pode-se concluir, portanto, que o fomento do $\mathrm{CNPq}$, dirigido a grandes áreas e áreas específicas, traz contribuições significativas às políticas governamentais. No entanto, deve-se destacar que, embora o desenvolvimento social seja considerado um dos objetivos da política científicotecnológica nacional, os temas sociais ou aqueles vinculados às Humanidades, áreas tradicionalmente responsáveis pelo seu desenvolvimento, tiveram pouco espaço nesse quadro. E está aí a diferença básica entre as áreas do conhecimento analisadas. Temas considerados "estratégicos" pelo CNPq obtiveram espaço na política científico-tecnológica nacional, privilegiando algumas áreas do conhecimento que convergem mais significativamente com tais temas. Por outro lado, áreas como as Ciências Humanas - e os temas que as tocam mais profundamente - praticamente não se encontram contemplados na mencionada política. Relacionam-se a temas menos prestigiados pela agenda governamental do CNPq e pelas diretrizes governamentais.

Percebe-se que os temas considerados "estratégicos" para o CNPq são aqueles que foram incluídos entre as prioridades temáticas do Plano Plurianual. ${ }^{5}$ Por outro lado, as áreas do conhecimento mais ligadas a esses temas, como as Ciências Biológicas e as Ciências da Saúde, são aquelas que mais transitam por diferentes temas e, por conseguinte, por diferentes disciplinas. Significa dizer que os temas definidos como prioritários pelo CNPq, aqueles que mais recebem fomento e mais aparecem no Plano Plurianual (PPA), estimulam pesquisas que transitam entre disciplinas distintas. Percebe-se, portanto, a relação entre a definição de temas prioritários e o desenvolvimento da multidisciplinaridade.

\section{A multidisciplinaridade nos cursos de pós-graduação da Capes}

A partir da década de 1990, observa-se o surgimento e o desenvolvimento de cursos multidisciplinares na pós-graduação, tendo sido especialmente estimulados por políticas elaboradas e implementadas pela Capes. A análise do gráfico abaixo revela a participação das diferentes áreas do conhecimento na pós-graduação brasileira durante o período que vai de 1987 a 2003. Constata-se a predominância das Ciências da Saúde frente às demais, apesar de se observar um leve recuo do seu crescimento no período mais recente. As demais áreas mantiveram-se relativamente estáveis, apesar da queda considerável do número de programas em

\footnotetext{
5 Embora o PPA-MCT tenha estabelecido prioridades temáticas através de programas para o período 2000-2003, as pesquisas auxiliadas pelo CNPq, em anos anteriores, através de bolsas de demanda espontânea (PIBIC, mestrado, doutorado e PQ), convergiram, em algumas áreas, com apenas parte dessas prioridades. Isso pode estar associado ao fato dos programas que obtiveram maiores níveis de convergência já serem objeto de ações de fomento do CNPq, há alguns anos, com participação da comunidade científica na sua elaboração. Embora outros programas do PPA também já fossem objeto de ações por parte do governo, essas não eram ações especificamente articuladas prioritariamente pelo CNPq.
} 
áreas como as Ciências Exatas e da Terra. Interessa aqui ressaltar o crescimento significativo de programas na área multidisciplinar, como indicado no gráfico abaixo.

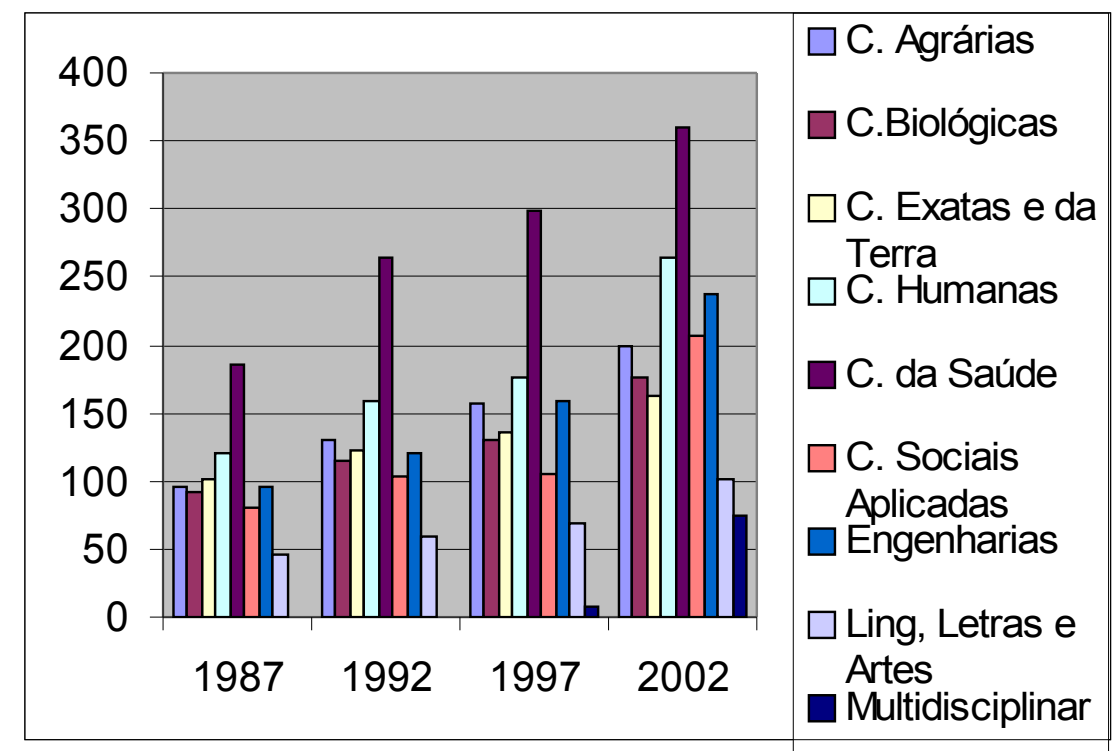

Figura 2: número de programas de pós-graduação por grandes áreas do conhecimento

A partir da análise do número de titulados no Mestrado e no Doutorado, percebe-se que a área multidisciplinar cresceu significativamente. O aumento do número de titulados no Mestrado foi de $5 \%$ em média, enquanto que o aumento relativo ao número de titulados no Doutorado foi de $1 \%$. Esse dado revela que o desenvolvimento dessa área é recente e, por essa razão, está se iniciando pela formação no Mestrado. Vale ressaltar que a baixa porcentagem apresentada mascara dados brutos relevantes. Enquanto em 1992 havia 10 titulados no mestrado na área multidisciplinar, em 2003 o número foi de 1.405 titulados. O mesmo pode ser observado entre os titulados no doutorado. De zero titulado no doutorado em 1992, 121 estudantes passam a integrar a categoria em 2003, como pode ser observado na figura a seguir.

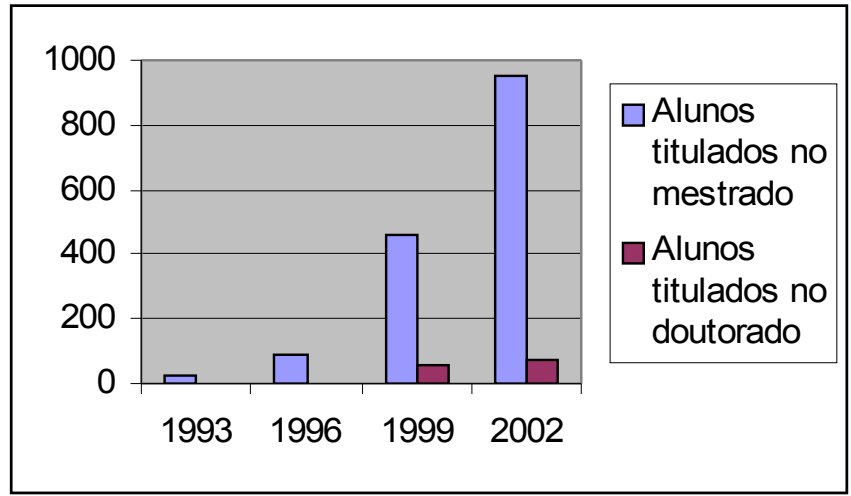

Figura 3: número de titulados no mestrado e no doutorado na área multidisciplinar 
Os dados que indicam a distribuição percentual dos docentes permanentes no mestrado e no doutorado no Brasil também revelam um fato curioso. Os cursos multidisciplinares, que em 1992 formavam os seus primeiros titulados no mestrado, crescem de forma significativa, representando, atualmente, $5,1 \%$ do total de docentes atuantes no Brasil. Vale ressaltar a extrema proximidade com o percentual encontrado na área de Lingüística, Letras e Artes, áreas do conhecimento já estabelecidas há várias décadas no Brasil. É curioso perceber, também, o crescimento e consolidação da área de estudos multidisciplinares, uma área relativamente nova que, quando comparada à de Lingüística, apresenta percentual semelhante de docentes envolvidos com os temas e objetos de estudo da área. Ao observar a evolução da área multidisciplinar separadamente, percebe-se de forma clara a sua expansão. Em 1987, a área apresentava 08 docentes permanentes no mestrado e no doutorado. Em 2002, 1.432 docentes atuaram nesse campo, como mostra a tabela abaixo.

\begin{tabular}{|c|c|c|c|c|c|}
\hline \multirow{2}{*}{$\begin{array}{c}\text { Grandes } \\
\text { áreas }\end{array}$} & \multirow{2}{*}{ Anos } & \multicolumn{2}{|c|}{ Número de cursos } & \multicolumn{2}{|c|}{ Docentes permanentes $(1)$} \\
\hline & & Mestrado & Doutorado & Total & Doutores \\
\hline \multicolumn{6}{|c|}{ Multidisciplinar } \\
\hline & 1987 & 1 & - & 8 & 8 \\
\hline & 1988 & 2 & - & 22 & 15 \\
\hline & 1989 & 2 & - & 24 & 17 \\
\hline & 1990 & 3 & - & 32 & 25 \\
\hline & 1991 & 3 & - & 33 & 26 \\
\hline & 1992 & 4 & - & 40 & 31 \\
\hline & 1993 & 5 & 1 & 52 & 40 \\
\hline & 1994 & 13 & 7 & 203 & 169 \\
\hline & 1995 & 19 & 9 & 319 & 268 \\
\hline & 1996 & 32 & 11 & 569 & 500 \\
\hline & 1997 & 39 & 12 & 734 & 667 \\
\hline & 1998 & 34 & 10 & 661 & 605 \\
\hline & 1999 & 50 & 13 & 973 & 925 \\
\hline & 2000 & 57 & 16 & 1.194 & 1.148 \\
\hline & 2001 & 73 & 19 & 1.457 & 1.407 \\
\hline & 2002 & 91 & 24 & 1.456 & 1.432 \\
\hline
\end{tabular}

Fonte: Coordenação de Aperfeiçoamento de Pessoal de Nível Superior (Capes) do Ministério da Educação (MEC). Elaboração: Coordenação-Geral de Indicadores - Ministério da Ciência e Tecnologia. 
Nota: A partir de 1997, passou-se a considerar docentes permanentes apenas aqueles com dedicação à pós-graduação de, no mínimo, $30 \%$ de sua carga horária.

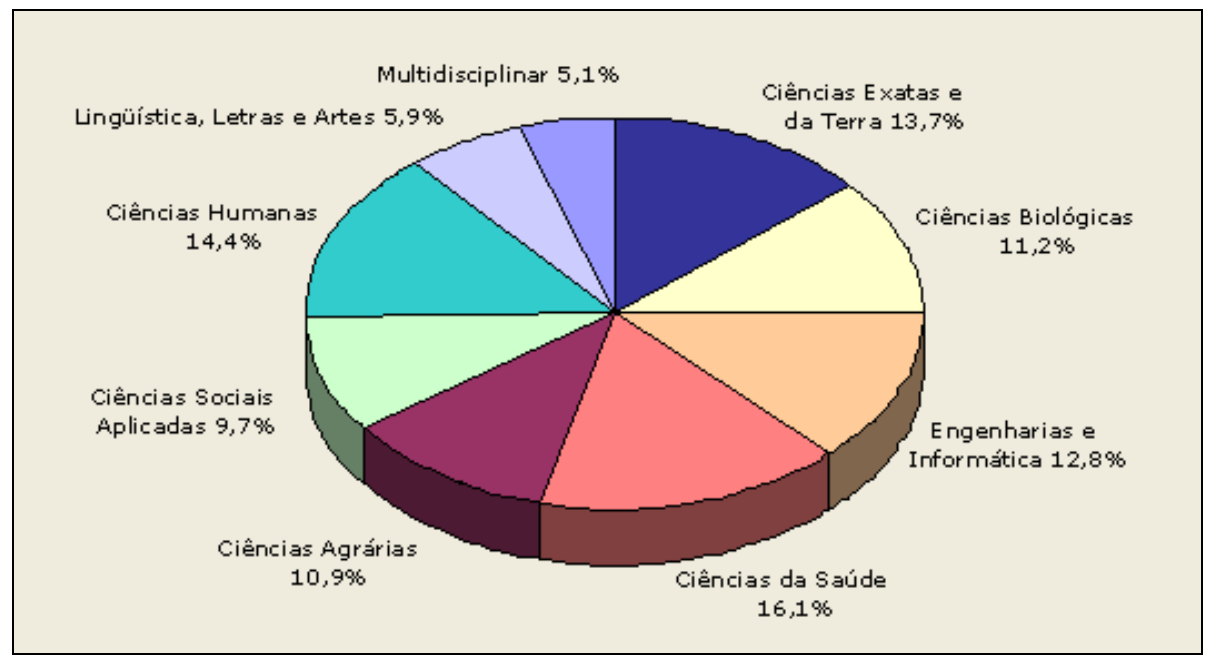

Figura 4: Brasil - Distribuição percentual dos docentes permanentes no mestrado e doutorado, por grandes áreas do conhecimento, 2002

Fonte: Coordenação de Aperfeiçoamento de Pessoal de Nível Superior (Capes) do Ministério da Educação (MEC). Elaboração: Coordenação-Geral de Indicadores - Ministério da Ciência e Tecnologia.

\section{Das disciplinas aos temas na política científica e tecnológica recente}

Quando são analisados alguns planos e programas do governo anterior e do atual, percebe-se um estímulo maior à pesquisa aplicada e tecnológica, à constituição de projetos cooperativos entre as universidades e outras instituições públicas e privadas e um estímulo, inclusive, à multidisciplinaridade.

São exemplos da política científico-tecnológica brasileira recente: o subprograma de Biotecnologia (SBIO 97); o subprograma de Ciências Ambientais (CIAMB de 1997); o Programa de Formação de Recursos Humanos em Áreas Estratégicas (RHAE de 1999); o programa de Apoio à Competitividade do Agronegócio (1998-2002) do CNPq e o Programa de Pesquisa em Saneamento Básico (PROSAB de 2000) da FINEP. Também o Plano Plurianual (PPA 2000-2003), os Fundos Setoriais e o novo programa "Institutos do Milênio" apresentam as características apontadas anteriormente. Vale ressaltar que constam dos objetivos do PPA 20002003:

- Consolidar, expandir e aprimorar a base nacional de ciência e tecnologia. 
- Viabilizar um efetivo sistema nacional de inovação; faz-se necessária, para tanto, a alocação de uma lei de inovação (em preparação), a fim de estimular as empresas privadas a investirem em inovação tecnológica.

- Preparar o país para os desafios da sociedade da informação e do conhecimento.

- Promover a capacitação científica e tecnológica em setores estratégicos para o desenvolvimento do país.

- Inserir a ciência e tecnologia nas estratégias de desenvolvimento social.

O Plano Plurianual 2000-2003 apresentava quatro programas estruturantes: Biotecnologia e Recursos Genéticos; Inovação para Competitividade; Sociedade da Informação e Internet II; Climatologia, Meteorologia e Hidrologia. Além desses quatro programas estruturantes, outros 15 programas temáticos também estão presentes no PPA/MCT, tais como os programas de Segurança Nuclear, Aplicações Nucleares na Área Médica, Ciência e Tecnologia para o Agronegócios, Atividades Espaciais, Ciência e Tecnologia para a Gestão de Ecossistemas, Desenvolvimento Tecnológico na Área Nuclear, Fomento ao Desenvolvimento Tecnológico no setor Petrolífero, Produção de Componentes e insumos para a Indústria Nuclear de alta Tecnologia, Fomento à Pesquisa em Saúde, Mudanças Climáticas e Produção de Equipamentos para a Indústria Pesada. Todos esses programas temáticos apresentam, além de um forte componente aplicado e tecnológico, a necessidade da presença de abordagens de diferentes disciplinas para o seu desenvolvimento.

Os Fundos Setoriais também podem apontar essas novas tendências. Além de representarem uma nova forma de financiamento para a ciência e, sobretudo, para a tecnologia, a iniciativa cria recursos estáveis e desvinculados do orçamento geral da União. Os recursos desses Fundos vêm de receitas fiscais extra-orçamentárias e do faturamento das empresas atuantes em importantes setores da economia. Essas receitas e outras formas de faturamento são utilizadas para financiar projetos de pesquisa nos setores dos quais as empresas fazem parte, como o de energia, petróleo, transportes, telecomunicações, mineração e espaço.

Por fim, existem os "Institutos do Milênio", sob a forma de redes de pesquisa que se formam segundo prioridades estabelecidas pelo Ministério da Ciência e Tecnologia (MCT). A inovação desse programa refere-se, em primeiro lugar, ao processo, pois pretende consolidar a articulação de redes de pesquisa. Em segundo lugar, inova quanto ao produto, pois o conhecimento deve contribuir para o aumento da competitividade da economia brasileira e para a resolução de grandes problemas nacionais de cunho social. Esses institutos estão divididos em dois grupos. Dois terços dos recursos financiam um primeiro grupo, que é aberto a todas as áreas de conhecimento, mas cujos objetivos devem estar voltados para a aplicabilidade. O restante dos recursos destina-se a um segundo grupo que deve atuar em áreas estratégicas, como o "Instituto do Milênio do Semi-Árido" - que desenvolve pesquisa nas áreas de Biodiversidade, Bioprospecção e Conservação de Recursos Naturais - e o "Instituto do Milênio de Oceanografia", voltado para o uso e a apropriação de recursos costeiros.

Há que se considerar, finalmente, o Plano Estratégico do Ministério da Ciência e Tecnologia (2004-2007) integrante do atual Plano Plurianual (PPA). Esse Plano é composto de três eixos verticais sobre um plano horizontal. O plano horizontal prevê a transformação de um sistema de ciência e tecnologia, hoje concentrado nas ações do Governo Federal e de alguns estados da Federação, em um sistema nacional de Ciência, Tecnologia e Inovação (CT\&I), com ampla participação de agentes públicos e privados presentes em todas as regiões e setores. Revela-se aí 
o estímulo das agendas governamentais à multidisciplinaridade e à heterogeneidade institucional no contexto de produção científico-tecnológica.

O primeiro eixo vertical vincula as atividades do Ministério da Ciência e Tecnologia (MCT) às prioridades das políticas industriais, tecnológicas e de comércio exterior. Para isso, foram identificados quatro setores estratégicos - software; fármacos e medicamentos; semicondutores e microeletrônica; bens de capital - e três áreas promissoras - a biotecnologia, a nanotecnologia e a biomassa.

O segundo eixo vertical inclui os objetivos estratégicos nacionais, nos quais são enfatizados o programa espacial, o programa nuclear, a Amazônia, o Cerrado, o Pantanal, o Semi-Árido e a plataforma marítima brasileira.

O terceiro eixo vertical é o da inclusão social que apóia programas e ações voltadas para a difusão científica e tecnológica. $O$ apoio vem sendo dado também à criação de centros vocacionais tecnológicos (CVTs), cujo objetivo é garantir emprego e renda nas regiões mais pobres do país. Nesse eixo, alguns programas se destacam, como o de Tecnologias Sociais, Arranjos Produtivos Locais, Biodiesel, Tecnologias de habitação, de Pesquisa em Saneamento Básico, de Incubadoras em Cooperativas Populares, de Pesquisa em Saúde, de Desenvolvimento Local, de pesquisa em Segurança Alimentar e Nutricional e Inclusão Digital. Sendo assim, podese perceber que algumas prioridades do Plano Plurianual anterior permanecem no Plano atual, como biotecnologia, saúde, programa espacial, nuclear e sociedade da informação, todos apresentando importância estratégica para o país.

Evidencia-se, assim, que as prioridades de fomento estão sendo consideradas a partir de problemas que exigem soluções ou de temas que demandam financiamento para pesquisa que é desenvolvida por diferentes áreas do conhecimento, e não por áreas disciplinares específicas e tomadas isoladamente. Ou seja, a multidisciplinariedade está se tornando cada vez mais presente nas agendas das instituições que estimulam a pesquisa científica e tecnológica.

\section{Conclusão: multidisciplinaridade e bens simbólicos}

As agendas governamentais, seus temas, intenções e financiamentos prioritários colaboram significativamente para as mudanças no campo de produção do conhecimento científicotecnológico. Uma dessas mudanças é a produção multidisciplinar de conhecimento. Foi aqui explicitado como isso acontece, como determinados estímulos e fomentos geram pesquisas com temas abrangentes, nada rigorosamente enquadrado dentro de apenas uma disciplina ou área do conhecimento. Tais características indicam novas práticas e relações no universo de produção científico-tecnológica, sendo a tendência à multidisciplinaridade e à heterogeneidade institucional os focos centrais. Foi observada também a interdependência maior entre diversos segmentos sociais ou, mais especificamente, entre as universidades, empresas e governo. Percebe-se, com isso, a formação de um novo cenário referente à produção de ciência, tecnologia e inovação no Brasil e no mundo.

As redes de produção de ciência e tecnologia passam a ser mais fluidas, pertencendo direta ou indiretamente à academia. Constituem redes não associadas, necessariamente, a um único campo social. São agentes que compõem vários cenários. Uma rede de produção de conhecimento pode ser hoje constituída de inúmeros atores, incluindo empresários e políticos. O cenário observado é 
caracterizado pela fluidez e flexibilidade presentes na formação e constituição das redes acadêmicas (FREITAS, 2004). Se esses indivíduos são intercambiáveis, as lógicas que regem cada um dos campos aos quais pertencem também são. Significa sugerir que mais importante que identificar lógicas distintas entre campos sociais ou entre subcampos - objetivo de teorias clássicas anteriores como a de Bourdieu (1994) - é analisar as interseções que geram essas teias complexas de relações e práticas. Não está sendo negada aqui a existência de bens simbólicos específicos de cada campo, mas apenas sugerida a importância de se analisar a tendência à intercambialidade constante entre lógicas distintas, tornando-as, na prática, indissociáveis umas das outras. Tendo em vista essa fluidez entre lógicas de campos distintos, a análise da existência de um conjunto de regras e princípios associados a um campo social particular, tomado isoladamente, torna-se questionável. A interseção observada não se dá apenas entre campos, como o econômico e o político, mas pode ser observada também no interior de cada área do conhecimento.

Não se pretende afirmar, aqui, a existência, no âmbito de todo o campo de produção da ciência, de características que necessariamente são identificadas em todos os grupos e contextos vividos no início do século XXI. Percebe-se isso ao analisar as diferentes áreas do conhecimento, seus diferentes perfis e níveis de inserção e participação em práticas consideradas "novas". Além disso, observa-se o constante entremear entre práticas e relações acadêmicas tradicionais e recentes. Sendo assim, não se pode dissociar, quase que estaticamente, um "novo modo" de um "modo anterior", já que ambos fundem-se constantemente na realidade observada. Ainda que tipologicamente tal dissociação pudesse ser realizada, interessou aqui não apenas apresentar uma e outra forma, mas analisar as implicações dessa fusão ou a interseção de características de lógicas disciplinares distintas.

A análise dos dados do fomento do $\mathrm{CNPq}$, dos cursos de pós-graduação da Capes e dos instrumentos de política científica e tecnológica recentes indica a tendência ao "modelo misto de desenvolvimento científico e tecnológico" apresentado. Nele, articulam-se a demanda espontânea e a demanda induzida, a comunidade científica, o governo e outros setores interessados no desenvolvimento científico e tecnológico. Esse modelo procura associar a lógica do campo científico, ou seja, as demandas da própria evolução da ciência às demandas econômicas e sociais, permitindo a articulação do campo científico ao campo econômico e social. Percebe-se, com isso, a fluidez de fronteiras entre disciplinas sem que as diferenças deixem de existir.

\section{Referências}

BOURDIEU, P. Algumas propriedades dos campos. In ORTIZ, R. (Org.). Pierre Bourdieu. São Paulo: Ática, 1994.

FREITAS, C. Práticas sociais no ciberespaço: novas redes de organização e circulação do conhecimento científico-tecnológico. Brasília, 2003. Tese (Doutorado em Sociologia) Departamento de Sociologia da Universidade de Brasília.

FREITAS, C. O capital tecnológico-informacional. Estudos de Sociologia, Recife: Revista do Programa de Pós-Graduação em Sociologia da UFPE, ano 7, 2004. 
GIBBONS, M. et al. The new production of knowledge. London: Sage, 1994.

NOWOTNY, H.; SCOTT, P.; GIBBONS, M. Re-thinking science: knowledge and the public in the age of uncertainty. Cambridge: Polity Press, 2001.

SOBRAL, F. A. F.; MARTINS, C. B.; VELLOSO, J. O fomento à pesquisa pelo CNPq e os programas temáticos prioritários do PPA/MCT. Brasília: Núcleo de Estudos sobre o Ensino Superior/UnB, 2003. Relatório de Pesquisa.

SANZ-CASADO, E. Study in interdisciplinarity in chemistry research based on the production of Puerto Rican scientists: 1992-2001. Information Research, [S.1.], v. 9, n. 4, jul. 2004. Disponível em: < http://informationr.net/ir/9-4/paper182.html >. Acesso em: ago. 2004.

SOBRAL, F. A. da F.; TRIGUEIRO, M. G. S. Limites e potencialidades da base técnicocientífica. In: FERNANDES, A. M.; SOBRAL, F. (Org.). Colapso da ciência e da tecnologia no Brasil. Rio de Janeiro: Relume Dumará, 1994. 